\title{
Evaluación del efecto de Trialeurodes vaporariorum (Hemiptera: Aleyrodidae) en el crecimiento de plántulas de Oreopanax floribundus (Araliaceae)
}

\author{
(D) Manuela Calderón-Hernández \\ Línea de investigación en Especies y Propagación, Sublínea en Conservación de Semillas, Subdirección Científica, \\ Jardín Botánico de Bogotá "José Celestino Mutis”, Bogotá, D.C., Colombia
}

\begin{abstract}
Resumen
Trialeurodes vaporariorum (Westwood) es un insecto fitófago que afecta diversos tipos de cultivos. En las labores del Jardín Botánico de Bogotá para la propagación masiva de especies nativas de bosque altoandino y páramo, se ha evidenciado la presencia de la mosca blanca. El objetivo del presente estudio fue documentar si Oreopanax floribundus podía ser una planta huésped de T. vaporariorum, y determinar si la presencia de este fitófago afectaba su crecimiento. El experimento incluyó tres tratamientos: T1 = control, T2 = plántulas con una infestación baja de T. vaporariorum, y T3 = plántulas con una infestación alta. El registro se realizó en dos momentos, al inicio del experimento ( $\mathrm{t} 0$ ) y dos meses después de la infestación (t1). Se registró la altura, el número de hojas y el peso seco en los dos momentos, así como la cantidad de ninfas en el t1 en hojas nuevas y viejas. Se encontraron diferencias entre el número de ninfas presentes en hojas nuevas y hojas viejas $(\mathrm{p}<0,05)$, pero no se encontraron diferencias entre los tratamientos en cuanto al crecimiento medido en peso seco y número de hojas $(p>0,05)$. Se presentaron diferencias $(\mathrm{p}<0,05)$ en la altura entre los tratamientos, siendo mayor el crecimiento con T2 y T3 que con T1. En conclusión, se aportó evidencia que permite catalogar a $O$. floribundus como una planta huésped de $T$. vaporariorum, y se abrió la posibilidad de estudiar con mayor profundidad si $O$. floribundus puede ser una planta tolerante a T. vaporariorum, lo cual es favorable para la propagacion masiva de esta especie. (C) 2018. Acad. Colomb. Cienc. Ex. Fis. Nat.
\end{abstract}

Palabras clave: Fitófago; Herbivoría; Mano de oso; Mosca blanca; Westwood.

Evaluation of the effect of Trialeurodes vaporariorum (Hemiptera: Aleyrodidae) on the growth of Oreopanax floribundus (Araliaceae) seedlings

\begin{abstract}
Trialeurodes vaporariorum (Westwood) is a phytopathic insect that affects different types of crops. The Botanical Garden of Bogota carries out the massive propagation of native species from the high Andean forest and páramo, in which the presence of whitefly has been evidenced. Our aim was to document if Oreopanax floribundus can be a host plant for T. vaporariorum and to determine if its presence affects the growth of the plant. The experiment consisted of three treatments: $\mathrm{T} 1=$ control, $\mathrm{T} 2=$ seedlings with a low infestation of $T$. vaporariorum, and $\mathrm{T} 3=$ seedlings with a high infestation. The record was made in two moments, at the beginning of the experiment $(\mathrm{t} 0)$ and two months after the infestation ( $\mathrm{t} 1$ ). The height, the number of leaves, and the dry weight were recorded in $\mathrm{t} 0$ and $\mathrm{t} 1$ and the number of nymphs in $\mathrm{tl}$ in new and old leaves. Differences were found between the number of immature flies present in new leaves and old leaves $(p<0,05)$, but no differences were found between treatments in growth measured in dry weight and number of leaves $(p>0,05)$. There were differences $(p<0,05)$ in the height between treatments where T2 and T3 showed a higher growth than T1. In conclusion, evidence was provided that can classify $O$. floribundus as a host plant of $T$. vaporariorum, and the opportunity opens up to further study if $O$. floribundus can be tolerant to $T$. vaporariorum, which is favorable for the massive propagation of this species. (C) 2018. Acad. Colomb. Cienc. Ex. Fis. Nat.
\end{abstract}

Key words: Mano de oso tree; Herbivory; Phytophagous; Westwood; Whitefly.

\section{Introducción}

La mayor limitante en la producción de cultivos en Colombia, como en muchas partes del mundo, son los insectos herbívoros, lo que causa pérdidas significativas a los agricultores y amenaza la seguridad alimentaria (FAO, 2017; Vilaseca, et al., 2008). La propagación de estos insectos ha aumentado drásticamente en los últimos años por diferentes causas, entre ellas el comercio del material vegetal (plantas, flores o frutos), el cambio climático y la poca capacidad de recuperación de los sistemas de producción debido a la intensificación de la agricultura durante años (FAO, 2017).

\footnotetext{
Correspondencia:

Manuela Calderón-Hernández, mchernandez@jbb.gov.co; macahek@hotmail.com Recibido: 25 de mayo de 2018

Aceptado: 29 de agosto de 2018

Editor: Marta Ramírez Pinilla
} 
Por ese motivo, y con el fin de generar las condiciones ambientales adecuadas para el desarrollo de las plantas cultivadas, se crean los invernaderos, los cuales, básicamente, son una estructura cubierta y cerrada que permite la entrada de luz solar y facilita la acumulación de calor durante el día, el cual se pierde lentamente durante la noche (FAO, 2002). Entre los beneficios que ofrece un invernadero se destaca el hecho de que permite cultivar y producir diferentes tipos de plantas, protegiéndolas de condiciones climáticas extremas, así como una producción continua, mejor calidad y reducción en los costos de producción al permitir un control y uso adecuado de plaguicidas, agua, fertilizantes y sustratos (Departamento Administrativo Nacional de EstadísticaDANE, 2014; Estrada, 2012). Sin embargo, según Monroy (2010), los invernaderos presentan condiciones adecuadas no solo para las plantas, sino también para la proliferación de una gran cantidad de insectos y enfermedades que pueden atacar los cultivos, por lo cual deben contar con programas para su manejo.

En diversos estudios se reporta cómo las moscas blancas se han convertido en unas de las plagas de mayor importancia para diferentes variedades de plantas silvestres y cultivadas en el mundo (Navarro, 2005). Las moscas blancas (Hemiptera: Aleyrodidae) son insectos fitófagos que ocasionan daños directos en las plantas, ya que en los estadios adulto e inmaduro se alimentan del floema de la planta succionando los fotoasimilados con sus estiletes (Rosell, et al., 1995), los cuales inserta en los tejidos de la hoja ocasionando ligeros daños físicos difíciles de cuantificar (Gullan \& Cranston, 2010), así como daños indirectos, ya que son vectores de virus (Navas-Castillo, $\boldsymbol{e t}$ al., 2011) y, además, promueven el crecimiento de hongos sobre el haz de la hoja de la plantas debido a la excreción de sustancias azucaradas, reduciendo la tasa fotosintética y causando la muerte prematura de los tejidos y estructuras (Carapia \& Castillo-Gutiérrez, 2013; López \& Segade, 2017). Mound \& Halsey (1978) reportaron más de 1.200 especies de moscas blancas en el mundo, sin embargo, los registros más comunes en Colombia, y los de mayor importancia económica, son Bemisia tabaci, Trialeurodes vaporariorum y Aleurotrachelus socialis (Navarro, 2005; Quintero, et al., 2001).

Trialeurodes vaporariorum es conocida como la mosca blanca de los invernaderos (Westwood) (Martin, 2005); su ciclo de vida dura, en promedio, 22 a 30 días a una temperatura promedio de $19{ }^{\circ} \mathrm{C}$ y una humedad relativa del 80 \% (Cardona, et al., 2005; Insecticide Resistance Action Committee-IRAC, 2017; Manzano \& Van Lenteren, 2009; Navarro, 2005). Se ha documentado que tiene cerca de 249 especies de plantas huéspedes (Quintero, et al., 2001; Vet, et al., 1980) distribuidas en 80 familias botánicas, de la cuales Astereaceae presenta el mayor registro, con 44 especies, seguida por Solanaceae (22) y Malvaceae (18) (Mound \& Halsey, 1978).
Esta lista de especies tiende a aumentar, y en algunos estudios se ha reportado la presencia de la mosca blanca en plantas silvestres aledañas a invernaderos; Roditakis (1990) reportó la presencia de la mosca blanca en 52 nuevas especies de plantas huéspedes en Creta y Grecia; en Kola, Rusia, Rak \& Litvinova (2010) registraron la plaga en las plantas de la colección viva del Jardín Botánico Kola y Ovčarenko, et al. (2016) reportaron cinco nuevas especies de plantas huéspedes en los alrededores de los invernaderos del oeste de Finlandia.

En los invernaderos del Jardín Botánico de Bogotá actualmente se realiza la propagación masiva de diferentes especies de plantas nativas y silvestres de los bosques altoandinos y de páramo, las cuales brindan diferentes servicios ecosistémicos pertinentes para la conservación, y durante las actividades de monitorización del crecimiento y la supervivencia del material propagado se reportó la presencia de $T$. vaporariorum en algunas de las especies.

Una de las plantas que se ha visto atacada por este fitófago es Oreopanax floribundus (Schult.) Decne. \& Planch (Araliaceae), un árbol nativo de Colombia (RiveraDíaz \& Celis, 2015) conocido comúnmente como mano de oso (Bernal, et al., 2017), especie de rápida germinación y crecimiento, usada comúnmente para la producción de combustible y también como especie ornamental en el arbolado urbano de Bogotá (Jardín Botánico de BogotáJBB, 2016), además de ser útil en la primeras etapas de restauración de ecosistemas degradados (Castillo, 2007). Aunque Mound \& Halsey (1978) reportaron a Aralia cordata y Oplopanax elatus, dos especies de la familia Araliaceae, como plantas huéspedes de T. vaporariorum, aún no hay reportes de que $O$. floribundus también pueda serlo. Por tal motivo, el objetivo de este estudio fue documentar si O. floribundus podía ser una planta huésped de T. vaporariorum $\mathrm{y}$, asimismo, determinar si su presencia afectaba el crecimiento de sus plántulas.

\section{Materiales y métodos}

El estudio se hizo en los invernaderos y laboratorios de la Subdirección Científica del Jardín Botánico de Bogotá (JBB), el cual está ubicado en la capital de Colombia (4³9'58.0”'N, 7405'56.4'O).

Material biológico. Las plántulas utilizadas en la investigación son el producto de las semillas de un individuo de O. floribundus que se encuentra en la colección viva del Jardín Botánico de Bogotá, las cuales fueron sembradas en julio del 2016 en un sustrato de tierra, humus y cascarilla en proporción de 50:30:20, respectivamente, y cuya edad era de un año y dos meses a partir de la siembra en el momento de comenzar el experimento. Cada planta se introdujo en una bolsa de plástico negro y se revisó para asegurarse de que no presentara patógenos o enfermedades antes de ser utilizada. Durante la evaluación del experimento las plantas no fueron fertilizadas y se las regó diariamente de manera uniforme humedeciendo directamente el sustrato sin mojar las hojas, para evitar la muerte de moscas adultas. 
La identificación taxonómica del fitófago de estudio se basó en la descripción morfológica comparativa de $B$. tabaci y T. vaporariorum propuesta por Carapia \& CastilloGutiérrez, (2013).

Se hizo una infestación dirigida con ninfas de los últimos estadios de desarrollo obtenidas de una cría de mosca blanca llevada a cabo en individuos de Solanum quitoense (planta huésped) en jaulas plásticas con un fotoperiodo de 12 horas, temperatura promedio de $17^{\circ} \mathrm{C}$ y una humedad relativa promedio de $74 \%$, valores que se tomaron con un Datalogger EBCHQ 94150 (China).

Evaluación experimental. El experimento se llevó a cabo en los meses de septiembre, octubre y noviembre de 2017; se utilizaron 90 plántulas con una altura promedio de $73 \mathrm{~mm}$, divididas en tres tratamientos (30 plantas), cada uno con tres réplicas (10 plantas).

Se construyeron nueve invernaderos pequeños (uno para cada réplica) de $32 \mathrm{~cm} \times 62 \mathrm{~cm}$ x $96 \mathrm{~cm}$ con canastas plásticas y palos de madera, recubiertos en su totalidad por plástico transparente para evitar la salida y entrada de las moscas o cualquier otro artrópodo; estos invernaderos, además, se aislaron en una casa de malla (Figura 1a).

El experimento consistió en comparar el crecimiento de plantas de $O$. floribundus expuestas a diferentes densidades de $T$. vaporariorum. Para esto se utilizaron tres tratamientos de la siguiente manera: el tratamiento uno, o de control, (T1), sin infestación y libre del fitófago; el tratamiento dos (T2), con una infestación baja (5 ninfas por planta), y el tratamiento tres (T3), con una infestación alta (200 ninfas por planta). Las plantas se infestaron cortando partes de hojas de $S$. quitoense que contaban con el número de ninfas
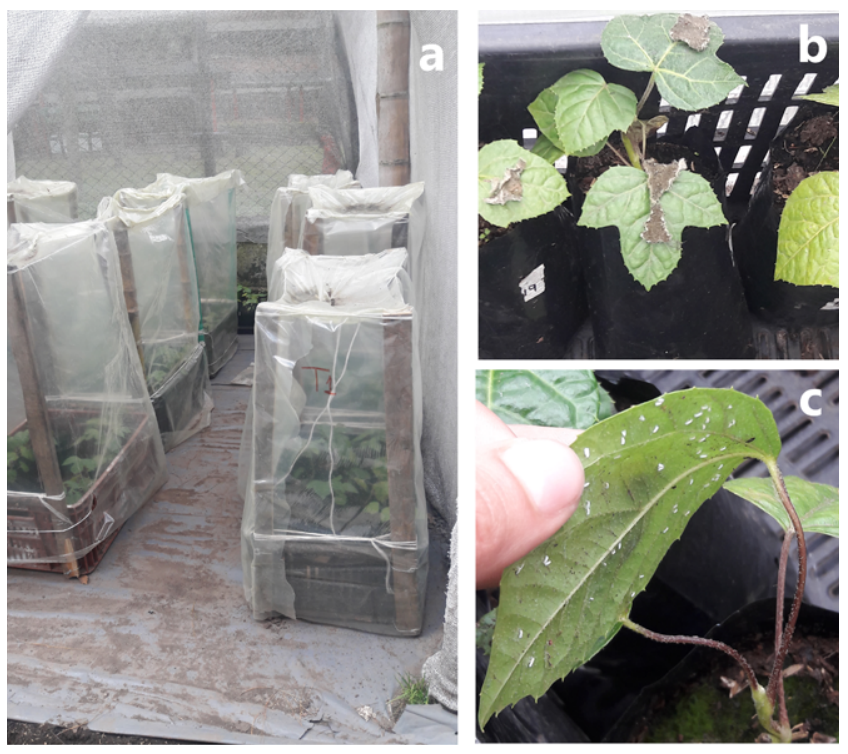

Figura 1. Etapas de desarrollo del experimento. a) Montaje de los invernaderos. b) Infestación con ninfas de T. vaporariorum de plantas de $O$. floribundus. c) Presencia de los primeros adultos después de la infestación en el tratamiento 3 deseadas en los estadios III o IV de desarrollo (Carapia \& Castillo-Gutiérrez, 2013), cuyos trozos se colocaron sobre las hojas de las plantas que se deseaban infestar (Figura 1b).

Los datos se registraron en dos momentos: al inicio del experimento, tiempo cero de evaluación (t0), y pasados dos meses de la infestación de las ninfas, tiempo 1 (t1), con el fin de no interferir en el desarrollo y el ciclo de vida de las moscas. A cada planta se le registró la altura y el número de hojas en el t0 y el t1, y la cantidad de ninfas presentes en el t1, diferenciando si se encontraban en hojas nuevas o viejas; a una muestra de cinco plantas por réplica se le calculó el peso seco total, el peso seco de la parte aérea y la parte terrestre en el t0 y en el t1, y a otras cinco plantas se les contaron la cantidad de tricomas presentes en el envés de las hojas nuevas y viejas antes de dividir los individuos por réplica. El recuento se hizo con ayuda de un estereoscopio Motic ${ }^{\circledR}$ SMZ-168 (Hong Kong, China), promediando en tres hojas por categoría la cantidad de tricomas presentes en dos cuadros de $1 \mathrm{~cm}^{2}$.

Análisis estadístico. A cada conjunto de datos a evaluar se le probó el supuesto de normalidad con la prueba de bondad de ajuste de Kolmogorov-Smirnov utilizando un intervalo de confianza del $95 \%$. Se hizo un análisis de varianza ANOVA simple, modelo I, también con un intervalo de confianza del $95 \%$, seguido del test de Tukey cuando fue necesario para evaluar si existían diferencias en: 1) el número de tricomas presentes en $O$. floribundus en hojas nuevas y en hojas viejas; 2) el número de ninfas reportadas en el t 1 en hojas nuevas y el número de ninfas en hojas viejas; 3) el número de ninfas reportadas en el t1 para los diferentes tratamientos, y 4) el peso seco, la altura y el número de hojas medidos en el t0 y el t1. Para los análisis se utilizó el programa estadístico StatGraphics ${ }^{\circledR}$ Centurion XVI, versión 16.1.11.

\section{Resultados y discusión}

Oreopanax floribundus puede ser una planta huésped para la mosca blanca $T$. vaporariorum, ya que se evidenció que después de mover ninfas criadas en una planta huésped conocida (S. quitoense) a una planta alternativa, en este caso la planta de estudio, los individuos aceptaron la planta alternativa como su huésped, lo cual se evidenció con la alimentación y la oviposición, que se toma como la deposición de uno o más huevos (Schoonhoven, et al., 2005), o la oviposición sostenida en el tiempo (César, et al., 2011). En este caso se evidenció la oviposición hasta de una segunda generación, ya que en el tiempo de evaluación t1 se encontraron ninfas de los últimos estadios, así como huevos recién puestos. Esta aceptación puede estar relacionada con el hecho de que T. vaporariorum es un insecto polífago y, tal como lo demostraron Tapia, et al. (2015) en su investigación, los generalistas poseen habilidades que les permiten aprender y hacer uso de nuevos huéspedes fácilmente.

Se encontraron diferencias en la densidad de los tricomas presentes en el envés de las hojas nuevas y viejas de $O$. floribundus $\left(\mathrm{F}_{1,9}=80,29 ; p<0,05\right)$, siendo significativamente 
más abundantes en las hojas nuevas (Figura 2a). Asimismo, se encontraron diferencias entre el número de ninfas pres-entes en las hojas nuevas y las hojas viejas tanto en el tratamiento dos $\left(\mathrm{F}_{1,59}=10,31 ; \mathrm{p}<0,05\right)$ como en el tratamiento tres $\left(\mathrm{F}_{1,59}=24,31 ; \mathrm{p}<0,05\right)$, dado que el mayor número de posturas se presentó en las hojas viejas que presentaban menor densidad de tricomas (Figura 2b-c). Estos resultados concuerdan con lo encontrado por Oriani \& Vendramim (2010) en diferentes genotipos de tomate, en los cuales encontraron que a mayor número de tricomas menor era el atractivo para la oviposición de la mosca blanca B. tabaco. Sin embargo, en el caso de la planta del algodón, Boiça Júnior, et al. (2007) encontraron que en las variedades estudiadas se presentó una mayor oviposición en genotipos de hojas pubescentes. Los tricomas son estructuras que pueden dificultar el movimiento y el comportamiento de la alimentación, especialmente de insectos pequeños, y se la ha reportado como una estrategia de resistencia constitutiva de las plantas para evitar el ataque de herbívoros (Schoonhoven, et al., 2005), sin embargo, no es la única variable a tener en cuenta para determinar la preferencia de un insecto por una planta. Es necesario profundizar en el papel de las toxinas o metabolitos secundarios que la planta puede producir y los mecanismos de defensa con los que cuenta el insecto (Boiça Júnior, et al., 2007), pues en los tejidos jóvenes, en general, se hallan mayores concentraciones de toxinas y antimetabolitos, lo que lleva a que los insectos polífagos prefieran tejidos más viejos, ya que en muchas ocasiones no cuentan con los mecanismos necesarios para asimilar y expulsar dichas toxinas (Cardona \& Mesa, 2011).

Se encontraron diferencias en el número de ninfas hallado en las plantas sometidas a los diferentes tratamientos $\left(\mathrm{F}_{2,87}=31,39 ; \mathrm{p}<0,05\right)$ (Figura 3), lo cual es importante, ya que de no haberlas encontrado no se podría comparar el efecto de la cantidad de individuos de mosca blanca en el crecimiento de la planta de estudio. Cabe resaltar que en la evaluación realizada en el t1 solo se contaron las ninfas que se encontraban entre los estadios II y IV y que correspondían a la primera generación de las ninfas introducidas en el t0, ya que, además del elevado número de adultos en el T3, se evidenció la presencia de un gran número de huevos e individuos de estadio I (Figura 1c).

En cuanto al crecimiento que presentaron las plantas con los diferentes tratamientos, no se encontraron diferencias en el peso seco total $\left(\mathrm{F}_{3,19}=1,72 ; \mathrm{p}=0,235\right)$, el peso seco de la parte aérea $\left(\mathrm{F}_{3,19}=2,6 ; \mathrm{p}=0,088\right)$ y el peso seco de la raíz $\left(\mathrm{F}_{3,19}=2,38 ; \mathrm{p}=0,107\right)$ de las plantas en el t0 y el $\mathrm{t} 1, \mathrm{y}$, aunque sí hubo un aumento del peso seco en el tiempo, este no se vio influenciado por la presencia de las moscas blancas (Figura 4). El peso seco es una de las variables más confiables para evaluar el crecimiento de una planta (Cardona \& Mesa, 2011; Suárez, et al., 2015), sin embargo, ya que las plantas con las cuales se trabajó hacen parte del activo bilógico del JBB, su número fue muy bajo, por lo cual este resultado debió complementarse con la altura y el número de hojas antes de plantear conclusiones.
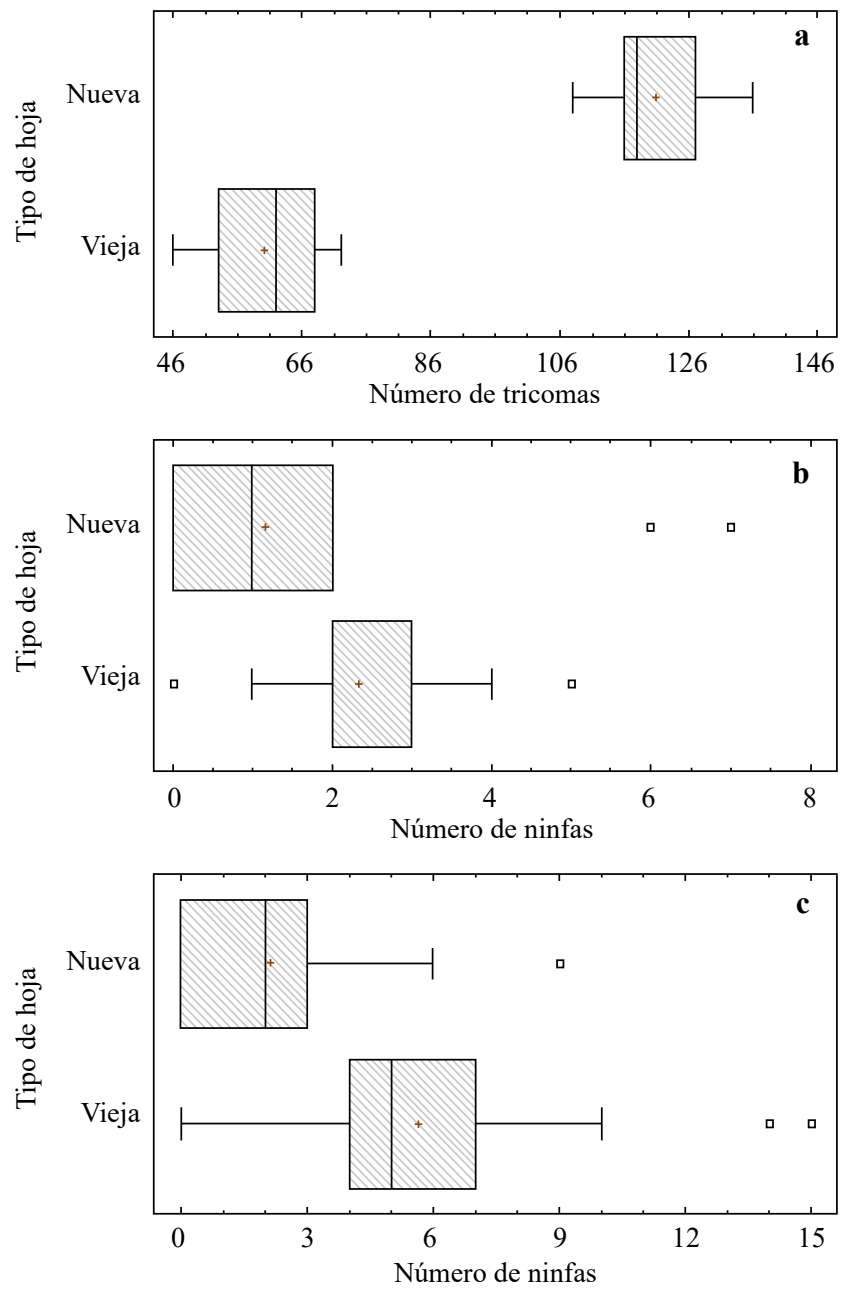

Figura 2. Tendencia central y dispersión de: a) número de tricomas en $O$. floribundus en hojas nuevas y viejas; b) número de ninfas de T. vaporariorum presentes en hojas nuevas y viejas con el T2; c) número de ninfas de $T$. vaporariorum presentes en hojas nuevas y viejas con el T3

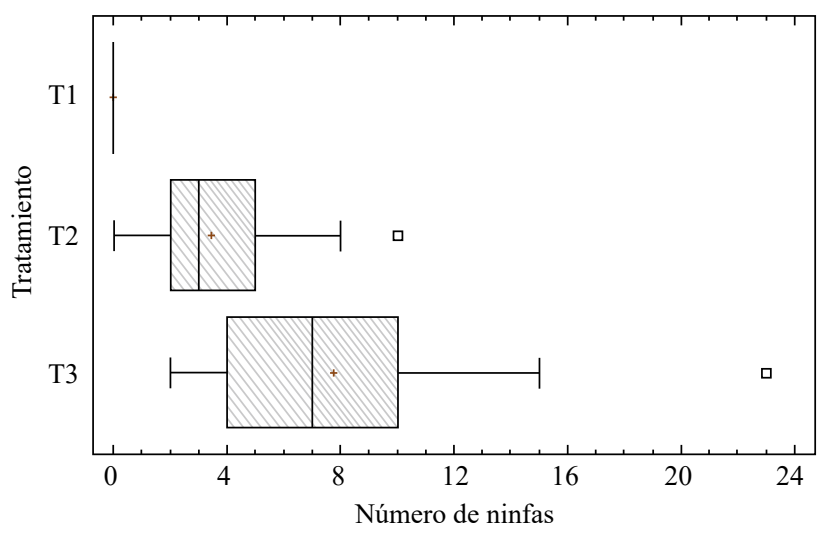

Figura 3. Tendencia central y dispersión del número de ninfas presentes en el tiempo de evaluación $\mathrm{t} 1$ (dos meses después de la infestación) con los diferentes tratamientos (T). T1: sin infestación, T2: baja infestación ( 5 ninfas por planta), T3: alta infestación (200 ninfas por planta) 


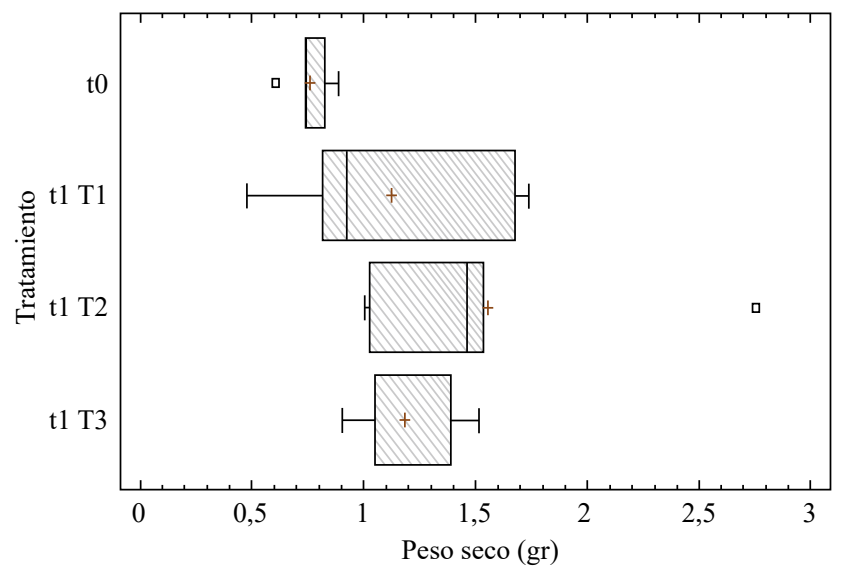

Figura 4. Tendencia central y dispersión del peso seco en plantas de $O$. floribundus sometidas a tres tratamientos $(\mathrm{T})$ con diferente grado de infestación de ninfas de $T$. vaporariorum evaluadas en el tiempo (t). t0: promedio del peso seco de las plantas en el tiempo inicial; $\mathrm{t} 1$ : dos meses después de la infestación; T1: sin infestación; T2: baja infestación ( 5 ninfas por planta); T3: alta infestación (200 ninfas por planta)

La altura y el número de hojas se compararon entre las plantas de cada tratamiento, no entre tratamientos, ya que se contaba con el registro de los individuos en el tiempo, lo cual permite evaluar mejor el cambio. En cuanto al número de hojas, no se presentaron diferencias en el T1 $\left(\mathrm{F}_{1,58}=0,99\right.$; $\mathrm{p}=0,32)$ ni en el T3 $\left(\mathrm{F}_{1,58}=0,14 ; \mathrm{p}=0,71\right)$, en tanto que en el T2 sí se encontraron diferencias $\left(\mathrm{F}_{1,58}=7,97 ; \mathrm{p}=0,006\right)$, al aumentar en una hoja, en promedio, durante el tiempo evaluado (Figura 5). Debe tenerse en cuenta que el mantener el mismo número de hojas a lo largo del tiempo también puede deberse a su recambio, ya que continuamente brotan nuevas hojas, al tiempo que se caen las más viejas. Sin embargo, los datos sustentan los resultados relacionados con el peso en el sentido de que el grado de infestación de la mosca blanca no afectó el desarrollo de las plantas.

Por último, en cuanto a la altura no se presentaron diferencias con el T1 $\left(\mathrm{F}_{1,58}=2,92 ; \mathrm{p}=0,09\right)$, pero sí con el $\mathrm{T} 2\left(\mathrm{~F}_{1,58}=21,21 ; \mathrm{p}<0,05\right)$ y el T3 $\left(\mathrm{F}_{1,58}=9,15 ; \mathrm{p}=0,003\right)$. En este sentido, se evidenció que la altura parece ser la variable más sensible al cambio en el crecimiento de las plantas a lo largo del tiempo, ya que en los tratamientos con infestación media y alta se presentó un mayor crecimiento que en el tratamiento control (Figura 6). Con estos resultados y en el marco del tiempo de evaluación del cambio en el crecimiento de las plantas de $O$. floribundus, se podría concluir que esta especie es tolerante a T. vaporariorum, entendiendo como tolerancia una respuesta expresada en la fisiología de los metabolitos primarios que le permite a la planta compensar el daño ocasionado por herbívoros (Schoonhoven, et al., 2005; Stout, 2013).

En conclusión este trabajó aportó evidencia que puede catalogar a O. floribundus como una planta hospedera de $T$. vaporariorum. Esta planta presenta una resistencia constitutiva que disminuye la oviposición en hojas nuevas, lo

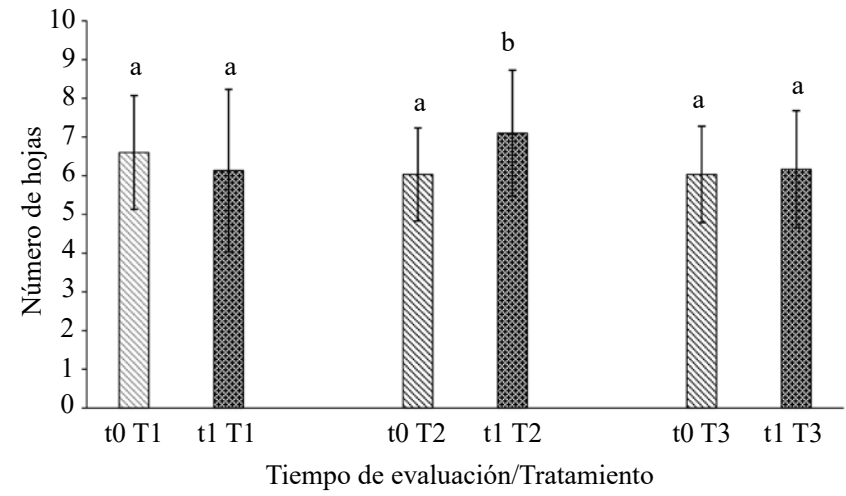

Figura 5. Número de hojas presentes en plantas de $O$. floribundus sometidas a tres tratamientos $(\mathrm{T})$ con diferente grado de infestación de ninfas de $T$. vaporariorum evaluadas en el tiempo (t). Las letras iguales indican que no hubo diferencias significativas en cada tratamiento según el Test de Tukey $(\mathrm{p}<0,05)$. Las líneas verticales indican la desviación estándar. t0: tiempo inicial; $\mathrm{t} 1$ : dos meses después de la infestación; T1: sin infestación; T2: baja infestación (5 ninfas por planta); T3: alta infestación (200 ninfas por planta)

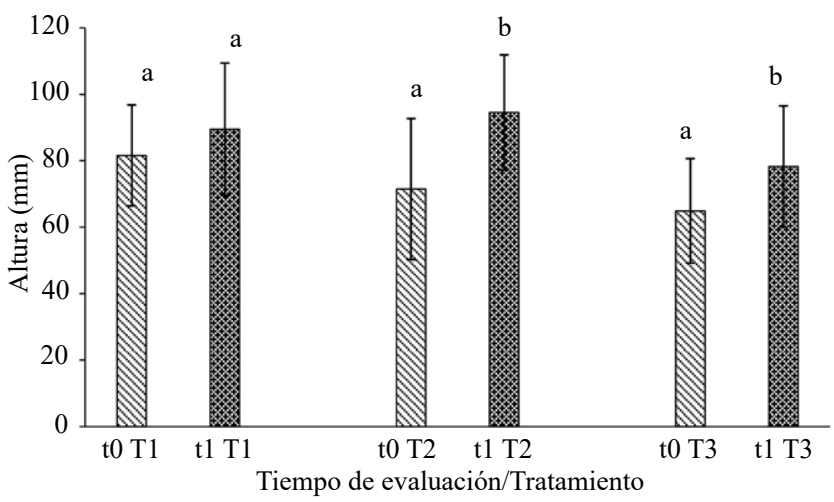

Figura 6. Altura de las plantas de $O$. floribundus sometidas a tres tratamientos $(\mathrm{T})$ con diferente grado de infestación de ninfas de $T$. vaporariorum evaluadas en el tiempo (t). Las letras iguales indican que no hubo diferencias significativas en cada tratamiento según el test de Tukey $(\mathrm{p}<0,05)$. Las líneas verticales indican la desviación estándar. t0: tiempo inicial; t1: dos meses después de la infestación; T1: sin infestación; T2: baja infestación ( 5 ninfas por planta); T3: alta infestación (200 ninfas por planta)

que lleva a este insecto hacer uso de las hojas viejas ya que presentan menos resistencia a la oviposición al presentar menos tricomas en el envés de la hoja. Queda planteada la necesidad de profundizar más en la posible tolerancia de $O$. floribundus a $T$. vaporariorum, lo cual es favorable para la propagacion masiva de esta especie, pues con niveles controlados de la plaga, se evitarían pérdidas del material propagado y podrían emplearse técnicas poco invasivas de control, como el uso de enemigos naturales.

\section{Agradecimientos}

Al Jardín Botánico de Bogotá por la financiación de la investigación; a la Dra. Helena Brochero, profesora de la 
Universidad Nacional de Colombia, sede Bogotá, y al ingeniero Álvaro Perdomo, investigador en fitosanidad del Jardín Botánico de Bogotá, por su asesoría en diferentes etapas de la investigación; al estudiante de biología John Sebastián Muñoz y a la operaria Ana Milena Torres por el apoyo en la recopilación de datos.

\section{Conflicto de intereses}

Los autores declaran no tener conflicto de intereses.

\section{Referencias}

Bernal, R., Galeano, G., Rodríguez, A., Sarmiento, H., Gutiérrez, M. (2017). Nombres Comunes de las Plantas de Colombia. Fecha de consulta: 24 de septiembre de 2018. Disponible en: http://www.biovirtual.unal.edu.co/ nombrescomunes/es/nombrescomunes/resultados/ncomun/ mano $\% 20 \mathrm{de} \% 20$ oso/

Boiça Júnior, A. L., Campos, Z. R., Lourenção, A. L., Campos, A. R. (2007). Adult attractiveness and oviposition preference of Bemisia tabaci (Genn.) (Homoptera: Aleyrodidae) B-biotype in cotton genotypes. Scientia Agricola, 64 (2): 147-151. https://doi.org/10.1590/S0103-90162007000200007

Carapia, V. E., \& Castillo-Gutiérrez, A. (2013). Estudio Comparativo sobre la Morfologia de Trialeurodes Vaporariorum (Westwood) y Bemisia Tabaci (Gennadius) (Hemiptera: Aleyrodidae). Acta Zoológica Mexicana. 29 (1): 178-193.

Cardona, C. \& Mesa, N. C. (2011). Resistencia varietal a insectos. Palmira: Universidad Nacional de Colombia, sede Palmira. p. 144

Cardona, C., Rodríguez, I., Bueno, J., Tapia, X. (2005). Biología y manejo de la mosca blanca Trialeurodes vaprariorum en habichuela y fríjol. Cali: Publicación CIAT. Fecha de consulta: 24 de septiembre de 2018. Disponible en: http:// books.google.com/books?hl=en\&lr=\&id=ZERwcoGwY24 $\mathrm{C} \&$ oi $=$ fnd\&pg $=$ PA $1 \& d q=$ Biología $+\mathrm{y}+$ Manejo + de + la + Mo sca+Blanca+Trialeurodes + vaporariorum + en + Habichuela + $\mathrm{y}+$ Fríjol\&ots=ElI5ajKop2\&sig=jp2tE3X3If7ob2SmKAtE rTjc0EA

Castillo, A. (2007). La investigación. Un aporte continuo del programa hojas verdes para la restauración ecológica del bosque andino en el Parque Ecológico la Poma. Fecha de consulta: 28 de agosto de 2017. Disponible en: http://www. caem.org.co/img/Castillo.pdf

Departamento Administrativo Nacional de Estadística-DANE. (2014). El cultivo del tomate de mesa bajo invernadero, tecnología que ofrece mayor producción, calidad e inocuidad del producto. Fecha de consulta: 28 de agosto de 2017. Disponible en: http://www.dane.gov.co/files/investigaciones/ agropecuario/sipsa/insumos_factores_de_produccion_ dic_2014.pdf

Estrada, J. J. P. (2012). Guía para la construcción de invernaderos o fitotoldos. FAO Bolivia. p. 80.

FAO. (2002). El cultivo protegido en clima mediterráneo. Estudio FAO, producción y protección vegetal, 90. Roma. Fecha de consulta: 28 de agosto de 2017. Disponible en: https://doi. org/ISBN 92-5-302719-3

FAO. (2017). Plagas y enfermedades de las plantas: FAO in Emergencies. Fecha de consulta: 26 de noviembre de 2017. Disponible en: from http://www.fao.org/emergencies/tiposde-peligros-y-de-emergencias/plagas-y-enfermedades-delas-plantas/es/
IRAC. (2017). Trialeurodes Vaporariorum. Fecha de consulta: 10 de octubre de 2017. Disponible en: http://www.irac-online.org/ pests/trialeurodes-vaporariorum/

JBB. (2016). Sistema de información para la gestión del arbolado urbano de Bogotá (Documento interno). Bogotá D.C.

López, S. N. \& Segade, G. (2017). Moscas blancas y enemigos naturales asociados al cultivo de naranja bajo dos tipos de manejo sanitario en San Pedro, provincia de Buenos Aires. Revista de La Sociedad Entomológica Argentina. 76: 11-21.

Manzano, M. R. \& van Lenteren, J. C. (2009). Life history parameters of Trialeurodes vaporariorum (Westwood) (Hemiptera: Aleyrodidae) at different environmental conditions on two bean cultivars. Neotropical Entomology. 38 (4): 452-458.

Martin, J. H. (2005). Whiteflies of Belize (Hemiptera: Aleyrodidae) Part 2-a review of the subfamily Aleyrodinae Westwood. Zootaxa. 1098: 1-116. Fecha de consulta: 10 de octubre de 2017. Disponible en: http://www.bio-nica.info/biblioteca/ Martin2005a.pdf

Monroy, L. M. R. (2010). Manejo de las plagas y enfermedades en invernaderos. Tecnoagro. 54. Fecha de consulta: 10 de octubre de 2017. Disponible en: https://tecnoagro.com.mx/ revista/2009/no-54/manejo-de-las-plagas-y-enfermedadesen-invernaderos/

Mound, L. \& Halsey, S. (1978). Whitefly of the world a systematic catalogue of the Aleyrodidae (Hómoptera) with host plant and natural enemy data. Richard Clay and Company Limited., Editors (William Cl). Great Britain. p. 340.

Navarro, V. R. (Editor). (2005). Manejo integrado de las moscas blancas. Bogotá, D.C Colombia: Produmedios. 41: 60.

Navas-Castillo, J., Fiallo-Olivé, E., Sánchez-Campos, S. (2011). Emerging Virus Diseases Transmitted by Whiteflies. Annual Review of Phytopathology. 49: 219-248. https://doi.org/10. 1146/annurev-phyto-072910-095235

Oriani, M. A. de G. \& Vendramim, J. D. (2010). Influence of trichomes on attractiveness and ovipositional preference of Bemisia tabaci (Genn.) B biotype (Hemiptera: Aleyrodidae) on tomato genotypes. Neotropical Entomology. 39 (6): 10021007. https://doi.org/10.1590/S0103-90162011000100006

Ovčarenko, I., Lindström, L., Saikkonen, K., Jauhiainen, L., Kaseva, J., Vänninen, I. (2016). Preconditioning of the generalist herbivore Trialeurodes vaporariorum to greenhouse monocultures and its subsequent performance on wild polycultures. Entomologia Experimentalis et Applicata. 159 (1): 1-16. https://doi.org/10.1111/eea.12428

Quintero, C., Rendón, F., García, J., Cardona, C., LópezAvila, A., Hernández, P. (2001). Especies y biotipos de moscas blancas (Homoptera: Aleyrodidae) en cultivos semestrales de Colombia y Ecuador. Revista Colombiana de Entomología. 27 (1-2): 27-31.

Rak, N. \& Litvinova, S. (2010). Hazardous organisms migration and acclimatization during the plant introduction in the far North Russia greenhouses. Hortus Botanicus. 5: 1-5. Fecha de consulta: 10 de octubre de 2017. Disponible en: http:// hb.karelia.ru

Rivera-Díaz, O. \& Celis, M. (2015). Oreopanax. En R. Bernal, S. Gradstein, \& M. Celis (Editores). Catálogo de plantas y líquenes de Colombia. Fecha de consulta: 10 de octubre de 2017. http://catalogoplantasdecolombia.unal.edu.co/es/ resultados/especie/Oreopanax\%20floribundus/ 
Roditakis, N. E. (1990). Host plants of greenhouse whitefly Trialeurodes vaporariorum Westwood (Homoptera: Aleyrodidae) in Crete. Attractiveness and impact on white-fly life stages. Agriculture, Ecosystems \& Environment. 31 (3): 217-224. https://doi.org/10.1016/0167-8809(90)90221-X

Rosell, R. C., Lichty, J. E., Brown, J. K. (1995). Ultrastructure of the mouthparts of adult sweetpotato whitefly, Bemisia tabaci Gennadius (Homoptera: Aleyrodidae). Int. J. Insect Morphol. \& Embryol. 24 (3): 297-306.

Schoonhoven, L., van Loon, J., Dicke, M. (2005). Insect-Plant Biology. Great Britain: Oxford University Press. p. 421.

Stout, M. J. (2013). Reevaluating the conceptual framework for applied research on host-plant resistance. Insect Science. 20 (3): 263-272. https://doi.org/10.1111/1744-7917.12011

Suárez, L., Díaz, M., Rodríguez, D., Cantor, F. (2015). Medición indirecta de la tasa de consumo de adultos e inmaduros de Trialeurodes vaporariorum (Hemiptera: Aleyrodidae) sobre fríjol. Acta Biológica Colombiana. 20 (3): 99-109. https://doi.org/10.15446/abc.v20n3.44073
Tapia, D. H., Silva, A. X., Ballesteros, G. I., Figueroa, C. C., Niemeyer, H. M., Ramírez, C. C. (2015). Differences in learning and memory of host plant features between specialist and generalist phytophagous insects. Animal Behaviour. 106: 1-10. https://doi.org/10.1016/j.anbehav. 2015.04.027

Vet, L. E. M., Lenteren, J. C., Woets, J. (1980). The parasitehost relationship between Encarsia formosa (Hymenoptera: Aphelinidae) and Trialeurodes vaporariorum (Homoptera: Aleyrodidae). Zeitschrift Für Angewandte Entomologie. 90 (1-5): 26-51. https://doi.org/10.1111/j.1439-0418.1980. tb03499.x

Vilaseca, C. J., Baptiste, L. G., López-Ávila, A. (2008). Incidencia de los márgenes sobre el control biológico natural de Spodoptera frugiperda (J. E. Smith) (Lepidoptera: Noctuidae) en cultivos de arroz. Corpoica- Ciencia y Tecnología Agropecuaria. 9 (2): 45-54. 